\title{
Correction to: Columbid circoviruses detected in free ranging pigeons from Southern Brazil: insights on PiCV evolution
}

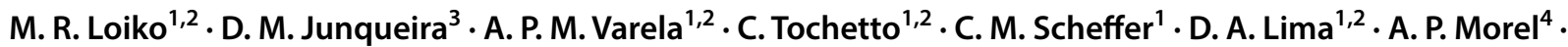 \\ C. Cerva $^{1,2} \cdot$ W. P. Paim ${ }^{1,2} \cdot$ Fabiana Quoos Mayer $^{2}$ (I) $\cdot$ P. M. Roehe ${ }^{1}$
}

Published online: 24 August 2018

C) Springer-Verlag GmbH Austria, part of Springer Nature 2018

\section{Correction to: Archives of Virology \\ https://doi.org/10.1007/s00705-018-3990-8}

Unfortunately, the word "evolution" was found missing in title of the original article which is corrected here by this erratum. The original article has been corrected.

The original article can be found online at https://doi.org/10.1007/ s00705-018-3990-8.

Fabiana Quoos Mayer

bimmayer@gmail.com

1 Laboratório de Virologia, Departamento de Microbiologia, Imunologia e Parasitologia, Instituto de Ciências Básicas da Saúde, UFRGS, Av. Sarmento Leite 500, sala 208, Porto Alegre, Rio Grande do Sul CEP 90050-170, Brazil

2 Laboratório de Biologia Molecular, Instituto de Pesquisas Veterinárias Desidério Finamor (IPVDF), Secretaria Estadual de Agricultura, Pecuária e Irrigação, Estrada Municipal do Conde, 6000, Eldorado do Sul, Rio Grande do Sul CEP 92990-000, Brazil

3 Centro Universitário Ritter dos Reis-UniRitter, Laureate International Universities, Porto Alegre, Rio Grande do Sul, Brazil

4 Falcoaria e Consultoria Ambiental-HAYABUSA, São Francisco de Paula, RS, Brazil 